\title{
Lorsque les médecins abusent des drogues*
}

II n'est pas rare qu'une discussion dans laquelle un confrère se confie puisse déboucher sur une prise de contact avec ReMed, le réseau de soutien pour les médecins. Mirjam Tanner, membre du Comité de direction de ReMed, nous décrit l'histoire exemplaire, et anonyme, d'un médecin confronté à une addiction [2] et montre les pistes proposées par ReMed pour en sortir.

Une chirurgienne expérimentée exerçant sous contrat avec un hôpital prend contact par messagerie électronique avec la ligne d'urgence de ReMed. Elle demande une prise de contact, non pas pour elle, mais pour un ami médecin-chef. Plus tard, au téléphone, elle déclare: «Cet ami m'a parlé de ses problèmes d'addiction. Il m'a confié que ses valeurs hépatiques étaient parfaitement normales alors qu'il consomme régulièrement de l'alcool fort. Ces cinq dernières années, il a augmenté sa consommation régulière de méthylphénidate. Le soir, sans alcool et sans Lexotanil, il n'arrive plus à 〈redescendre .» La chirurgienne voit dans le recours à tous ces produits stimulants un engrenage infernal. Elle comprend désormais mieux pourquoi elle avait l'impression que son confrère lui semblait avoir changé. Les remarques d'autres collègues sur son irritabilité, son impulsivité, son impatience croissantes à l'égard des patients pren- nent également une toute autre signification. Enfin, elle ajoute avoir pensé que tout ce qu'elle entendait relevait de la malveillance, que ces propos n'étaient que des rumeurs comme celle selon laquelle sa femme voulait le quitter ou l'avait déjà fait.

\section{ReMed: aide dans des situations de crise}

ReMed répond à toute prise de contact dans les 72 heures et examine la situation personnelle et les démarches individuelles possibles, sans engagement et en toute confidentialité, car la démarche est couverte par le secret médical. Avezvous besoin de soutien? Un médecin de votre entourage nécessite-t-il une aide?

N'hésitez pas à contacter ReMed 24h sur 24 par téléphone au $0800 \quad 0$ 73633, par courriel à info[at]swiss-remed.ch ou www.swiss-remed.ch.
* L'étude dite de Mayence du Prof. Klaus Lieb révèle qu'un chirurgien sur cinq recourt à des substances psychoactives légales ou illégales et que $15 \%$ affirment consommer des antidépresseurs. L'étude se fonde sur une nouvelle technique de questionnaire permettant de recueillir des réponses fidèles à ce qui a été vécu [1].

Correspondance: Dr Mirjam Tanner

mirjam.tanner[at]hin.ch

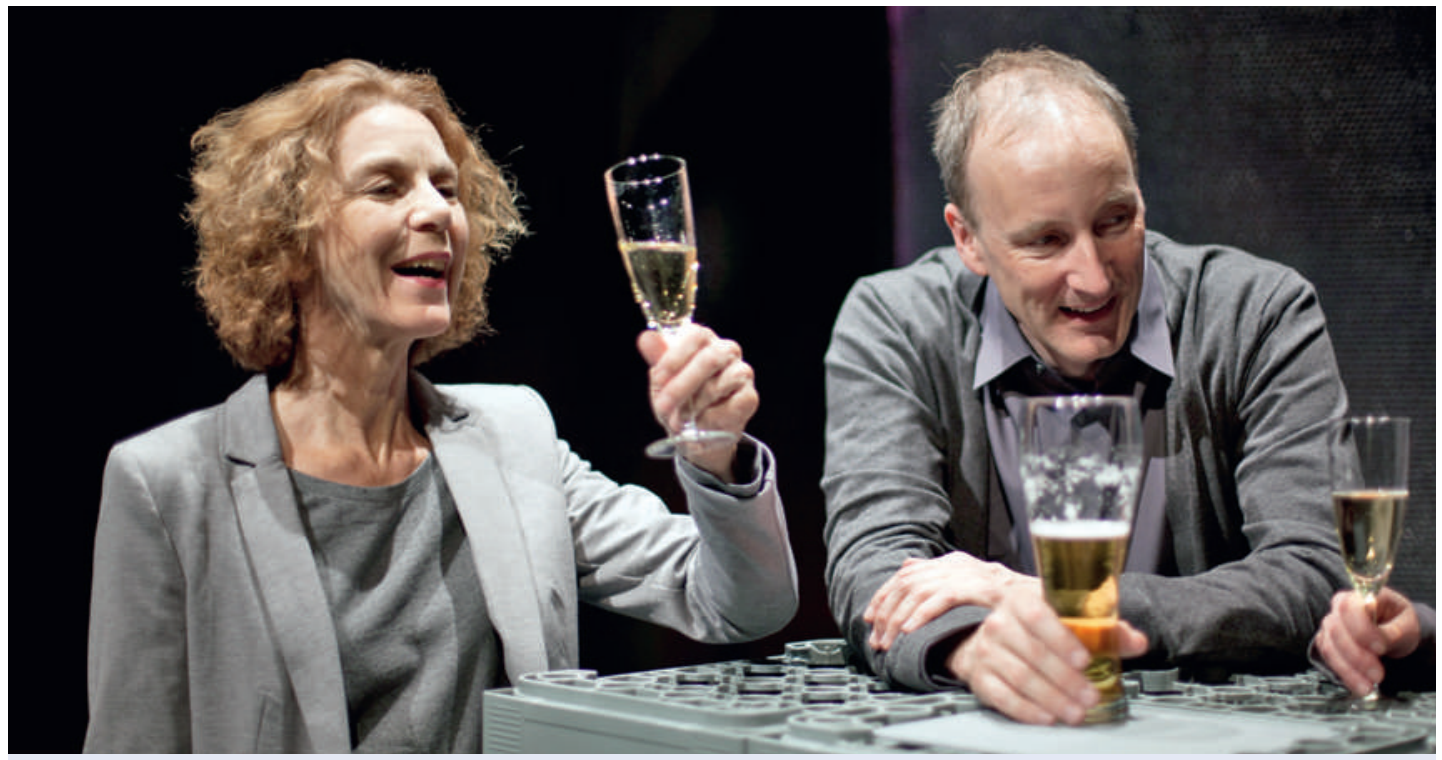

Briseur de tabou et libérateur: une scène du spectacle «A votre santé», une approche théâtrale interactive sur le rapport à l'alcool, mis en scène par le théâtre Knotenpunkt (www.theater-knotenpunkt.ch). ReMed se ferait un plaisir d'organiser une représentation interactive pour votre société de discipline, votre réseau de soins ou pour une session de formation continue à l'hôpital. Contact: j.baenninger[at]hin.ch 


\section{Honte, peur, solitude}

Notre interlocutrice souhaite savoir ce que ReMed conseillerait et entreprendrait dans une telle situation? L'anonymat de son collègue resterait-il préservé s'il prenait lui-même contact avec ReMed? «Il me semble profondément désespéré et je crains qu'il soit très seul», insiste-t-elle. Je confirme que les médecins sont souvent seuls face à leurs problèmes. Ils ne sont pas vraiment habitués à solliciter de l'aide pour euxmêmes. Lorsqu'il s'agit de dépendances, diverses peurs s'ajoutent souvent à la honte qu'ils éprouvent, notamment la crainte de perdre leur autorisation d'exercer s'ils avouent leur problème. Ainsi, nombreux sont les confrères qui préfèrent traverser seuls ces phases critiques de la vie et qui s'autoprescrivent des psychostimulants ou consomment des drogues.

\section{La confiance passe par le respect}

Nous regardons qui serait le mieux à même d'épauler notre confrère face à ses problèmes d'addiction. A-t-il

\section{Groupes d'intervision: les dates 2014}

En 2009, ReMed a initié des intervisions entre confrères, également pour répondre à un souhait exprimé. Aujourd'hui, le réseau de soutien organise régulièrement des sessions entre pairs (6-10 participants, 2-3 fois/an). Les participants abordent ensemble les questions de mentorat, de coaching, de conseil, de traitement mais aussi d'autres aspects de l'accompagnement collégial (droit, droit des assurances, etc.). Mettezvous en relation avec nous, participez à une de nos séances et découvrez notre travail. Contact: Peter Birchler, tél. 0443420910 ou peter. birchler[at]hin.ch. Dates encore disponibles en 2014: les 18 septembre, 30 octobre et 20 novembre à Zurich et le 6 novembre à Berne. un médecin de famille à qui se confier, ou serait-il disposé à en chercher un? Bien évidemment, il peut aussi s'adresser directement à ReMed. Là aussi, son anonymat serait parfaitement respecté. Il suffit de s'adresser à un des conseillers ReMed, qui analysera avec lui la situation en profondeur. Ensemble, ils chercheront la voie la mieux adaptée pour tourner le dos à la dépendance. Notre chirurgienne décide finalement de faire part de notre entretien à son confrère et de lui donner l'adresse e-mail et le numéro de téléphone d'un conseiller ReMed.

Le premier entretien consiste principalement à créer un climat de confiance dans lequel notre confrère se sente à l'aise pour s'ouvrir et nous raconter les raisons de sa démarche. Une fois rassuré qu'aucune dénonciation n'aura lieu de notre part et conscient du respect que nous lui accordons, il a commencé à nous dépeindre l'ampleur du problème lié à sa consommation d'alcool et de médicaments et à décrire ce sentiment de désespoir qui l'habite. Suite à une question du conseiller, il confirme: «Je n'attendrais jamais d'un patient qu'il arrive à surmonter lui-même une telle crise.» $\mathrm{Au}$ final, le médecin accepte de convenir d'un rendez-vous. Il est soulagé de pouvoir saisir cette perche qui lui est tendue, de s'occuper enfin de lui et de réfléchir sur le soutien professionnel qui pourrait l'aider à se libérer de ses dépendances.

\section{Références}

1 Doping auch am Skalpell - jeder fünfte Chirurg greift zu leistungssteigernden Substanzen, Medscape Deutschland. Dr. Erentraud Hömberg, www.medscapemedizin.de/artikel/4901231m (3 juillet 2013, en allemand)

2 Ott R, Biller-Andorno N. La signification du neuroenhancement dans la pratique médicale. Bull Méd Suisses. 2013; 94(13/14):504-6. 